Running head: Varenicline, cognitive bias modification and fMRI in smokers

\title{
Effects of varenicline and cognitive bias modification on neural response to smoking-related cues: a randomised control trial
}

\author{
Angela S. Attwood¹, 2, 3 , Tim M. Williams ${ }^{4}$, F. Joseph McClernon ${ }^{5}$, Rachel Kozink ${ }^{5}$, \\ Sally Adams ${ }^{6}$, Karl Scheeres ${ }^{7}$, Amy Green $^{4}$, David Christmas ${ }^{8}$, \\ Marcus R. Munafò̀ ${ }^{1,2,3}$
}

\footnotetext{
${ }^{1}$ MRC Integrative Epidemiology Unit at the University of Bristol, United Kingdom

2 UK Centre for Tobacco and Alcohol Studies, University of Bristol, United Kingdom

${ }^{3}$ School of Psychological Science, University of Bristol, United Kingdom

${ }^{4}$ Population Health Sciences, University of Bristol, United Kingdom

${ }^{5}$ Psychiatry and Behavioral Sciences, Duke University School of Medicine, USA

${ }^{6}$ Department of Psychology, University of Bath, United Kingdom

${ }^{7}$ Avon and Wiltshire Mental Health Partnership NHS Trust, United Kingdom

${ }^{8}$ Cambridge and Peterborough NHS Foundation Trust, United Kingdom
}

\section{${ }^{*}$ Corresponding author:}

Angela S. Attwood PhD, School of Psychological Science, University of Bristol, 12a Priory Road, Bristol BS8 1TU, United Kingdom. T: +44.117.331.7450; F:

+44.117.928.8588; E: angela.attwood@bristol.ac.uk

Author email addresses: Tim Williams - tim.williams6@nhs.net, F. Joseph McClernon francis.mcclernon@duke.edu, Rachel Kozink - rachel.kozink@duke.edu, Sally Adams -s.adams@bath.ac.uk, Karl Scheeres-karlscheeres@nhs.net, Amy Green amy.green@bristol.ac.uk, David Christmas - david.christmas@cpft.nhs.uk, Marcus R. Munafò - marcus.munafo@bristol.ac.uk. 

available under aCC-BY 4.0 International license.

Running head: Varenicline, cognitive bias modification and fMRI in smokers

35

36 Word count: 5604 (excl. abstract, tables, figures, references).

37

38 Key words: smoking, cognitive bias modification, cue reactivity, attentional bias,

39 relapse, fMRI.

40

41 Trial Registration: Current Controlled Trials ISRCTN65690030. Date of registration:

4230 January 2014.

43 
Running head: Varenicline, cognitive bias modification and fMRI in smokers

\section{Abstract}

Drug-related cognitive biases have been positively associated with drug-craving and increased likelihood of relapse. Cognitive bias modification paradigms have been developed to attenuate cognitive biases but there have been few studies that examined neural responses to these paradigms. This study compared neural responses following $\mathrm{CBM}$ and explored whether $\mathrm{CBM}$ effects were potentiated by varenicline administration. This was a double-blind placebo-controlled study with two between subject factors of drug (varenicline, placebo) and CBM (attend towards smoking cues, train away from smoking cues, control training) that recruited daily ( $\geq 10$ cigarettes per day) non-treatment seeking smokers. Participants ( $n=67,53 \%$ female) were randomised to one-week of drug administration (varenicline or placebo) before attending a study session at which they were randomised to CBM condition, and underwent an $\mathrm{fMRI}$ scan were they were presented with smoking and neutral cues. Neural response to smoking (vs. neutral) cues, cognitive bias, craving and mood were assessed. There was no evidence of CBM effects on any outcomes. There was evidence of effects of varenicline on craving, with greater reductions in craving in the week preceding the study session in the varencline group $\left(p=0.04, \eta_{p}^{2}=.06\right)$. There was also evidence of a drug by CBM interaction for neural responses $(z=3.78, p$ $<0.001)$. Compared to placebo, varenicline was associated with greater activation in the right temporal middle gyrus in the CBM control condition, compared to an opposite 64 effect in the CBM "attend towards" condition. These data suggest that CBM does not modify cognitive bias, subjective craving and mood, or neural response to smoking cues. There was also no evidence that CBM effects were potentiated by varenicline. 
Running head: Varenicline, cognitive bias modification and fMRI in smokers

\section{Introduction}

Smoking remains the leading cause of preventable death worldwide, with an estimated 6 million tobacco-related deaths occurring every year (World Health

Organization, 2013). Despite many smokers reporting wanting to quit, few achieve long-term abstinence. This may be partly due to the presence of smoking-related cues in the environment, which through repeated and contingent pairing with drug administration, acquire powerful motivational properties that can precipitate craving and drug seeking (Foltin \& Haney, 2000; Gray, LaRowe, \& Upadhyaya, 2008; McClernon et al., 2016; Mucha, Pauli, \& Angrilli, 1998; Muntaner et al., 1989). Drug-related cognitive biases, characterised by selective or disproportionate attention allocation to drug cues, have been reported in users of a number of drugs and have been positively associated with drug craving (Field, Munafo, \& Franken, 2009; Wakefield, Germain, \& Henriksen, 2008), future drug use (Cox, Pothos, \& Hosier, 2007), approach behaviours to drug-related cues (Franken, 2003) and increased likelihood of relapse (Marissen, Franken, Blanken, van den Brink, \& Hendriks, 2007). Of particular importance, the drug-stimulus learning that is believed to underlie these biases is long-lasting, which makes an individual vulnerable to relapse long after initial cessation. In smokers, increased reactivity to smoking cues has been found to predict decreased likelihood of cessation (Abrams, Monti, Carey, Pinto, \& Jacobus, 1988; Niaura, Abrams, Demuth, Pinto, \& Monti, 1989; Waters et al., 2004). Consequently, reduction in cognitive bias is a potential target for therapeutic intervention.

There is evidence that it is possible to reduce cognitive biases using computerbased cognitive bias modification (CBM) paradigms that "train" individuals to allocate attention away from disorder-relevant cues. CBM has been shown to reduce cognitive and has also been associated with reduction in other symptoms such as low mood (Baert, De Raedt, Schacht, \& Koster, 2010). Attwood and colleagues (Attwood, O'Sullivan, Leonards, Mackintosh, \& Munafo, 2008) reported decreased cognitive bias 
Running head: Varenicline, cognitive bias modification and fMRI in smokers

in a group of smokers following one session of stimulus-avoidance CBM using a modified dot probe task. Compared to a group who had been trained to attend to smoking cues, there was evidence that the avoid group also showed attenuated craving in response to in vivo smoking cues in a subsequent cue exposure test (male participants only). A subsequent study in tobacco smokers found similar decreases in cognitive bias following CBM, but did not observe generalisation of these effects of other relevant behaviours (e.g., cigarette craving) or novel (untrained) stimuli (Field, Duka, Tyler, \& Schoenmakers, 2009). The weak effects may be due to the limited number of sessions used in laboratory-based studies and that multiple session approaches may be more efficacious than single session CBM (Lopes, Pires, \& Bizarro, 2014; McHugh, Murray, Hearon, Calkins, \& Otto, 2010; Unrod et al., 2014). Taken together, the research suggests CBM may have some clinical utility but the effects are compromised by small effect sizes and issues with generalisation of the training effects.

There has been growing interest in the development of combination drugbehavioural therapies, in which a drug is used to augment the outcomes of a behavioural intervention (Swerdlow, 2012). This may offer a solution to the low efficacy and reliability of CET effects, if a suitable pharmacological agent can be identified. The smoking cessation pharmacotherapy varenicline acts as a partial agonist of the $\alpha 4 \beta 2$ nicotinic acetylcholine receptor and aids cessation by reducing cigarette craving and withdrawal symptoms. However, it has also been associated with a reduction in cuerelated craving in humans (Ray et al., 2013), particularly following chronic administration (Brandon et al., 2011), and reductions in cue-induced reinstatement of drug taking in animals (Le Foll et al., 2012; Wouda et al., 2011). Therefore, varenciline may be a useful adjunct to CBM, particularly as it is already licensed as a smoking cessation aid.

The current study replicated earlier work by examining the effects of CBM on behavioural measures of cognitive bias (visual dot probe and modified Stroop), and 
Running head: Varenicline, cognitive bias modification and fMRI in smokers

123 extended the work in two important ways. First, we examined whether 7-day pre-

124 treatment of varenicline enhanced the effects of CET on smoking cue reactivity and

125 attentional bias. Second, using $\mathrm{fMRI}$, we examined the neural responses to smoking

126 cues following treatment. Neuroimaging studies suggest that drug-related cognitive

127 biases are the result of a failure of cognitive regulatory systems to increase control in

128 the presence of salient cues that increase processing in the reward and emotional

129 centres of the brain (e.g., striatum, amygdala) (Hester \& Luijten, 2013). Therefore,

130 these additional measures offer important insight into the mechanisms underlying the

131 effects of CBM, particularly as computer-based measures of cognitive bias are known

132 to lack reliability (Ataya et al., 2012; Field \& Christiansen, 2012).

133 In this study, participants attended two sessions, approximately one-week

134 apart. Participants were randomised to receive either 7-day treatment of varenicline or matched placebo, prior to completion of 1-hour of CBM training. For CBM training,

136 participants were further randomised (stratified by drug group) to one of three

137 conditions: 1) training towards smoking cues (attend), 2) training away from smoking

138 cues (avoid), or 3) control training (control). After training, participants underwent a cue

139 reactivity task during an $\mathrm{AMRI}$ scan. We hypothesised that participants in the CBM-

140 avoid condition would show a decrease in cognitive bias and neural response to

141 smoking-related cues compared to those in the CBM-attend and CBM-control

142 conditions, and that changes in cognitive bias and neural response, would be greatest

143 in individuals trained to avoid smoking-related cues and treated with varenicline.

\section{Methods}

\section{Participants}

147 Daily smokers ( $\geq 10$ cigarettes or 15 roll ups/day who smoke within one hour

148 of waking) were recruited from the staff and students at the University of Bristol and

149 the general population through existing participant databases, posters, online flyers

150 and word of mouth. Participants were required to be between 18 and 40 years of 
Running head: Varenicline, cognitive bias modification and fMRI in smokers

151

152

153

154

155

156

157

158

159

160

161

162

163

164

165

166

167

168

170

171

172

age, fluent in English and registered with a General Practitioner. Exclusion criteria

were pregnancy, breast feeding or at risk of pregnancy (i.e., females not using

adequate contraception), substance misuse, high alcohol consumption (>35

units/week if female or $>50$ units/week if male) or caffeine consumption ( $>8$ cups of caffeinated beverage per day), current or past psychiatric disorder, clinically significant abnormality (including cardiology risk factors), use of medication (participants were required to be 8 weeks clear of any prescribed medication), known hypersensitivity to varenicline, high blood pressure or heart rate (systolic/diastolic $>140 / 90 \mathrm{mmHg}$ or heart rate $>90 \mathrm{bpm}$ ), uncorrected visual or auditory impairment, and any condition that would make MRI scanning unsafe (e.g., metallic implants) or intolerable. The study was approved by the National Research Ethics Service (London Brent Committee, reference: 11/LO/1726). All participants gave written informed consent and were reimbursed $£ 70$ at the end of the study.

\section{Design}

This was a double-blind, placebo-controlled study that used a $2 \times 3$ betweensubjects design, comprising one factor of drug (varenicline, placebo) and one factor of CBM group (attend, avoid, control). For the behavioural assessments of cognitive bias (dot probe, modified Stroop), there was an additional within-subjects factor of cue type (smoking, neutral).

\section{Drug administration}

Following initial consent and screening on day 0 , varenicline (or matched placebo) was prescribed by a medical doctor for one week. Participants were told to take one tablet $(0.5 \mathrm{mg})$ daily on days 1 - 3, two tablets daily (total $1 \mathrm{mg}$ ) on days 4 6 , and one tablet $(0.5 \mathrm{mg})$ on day 7 , consistent with standard dosing regimen for smoking cessation. Participants completed daily diaries detailing the time at which the tablets were taken and any side effects. Participants attended their second 
Running head: Varenicline, cognitive bias modification and fMRI in smokers

179 session on day 7 (i.e., their last drug day) and were asked to take the drug in the

180 morning prior to their study session.

\section{Randomisation}

Participants were randomly assigned to drug and CBM groups (stratified by gender), but equal numbers of participants per group were maintained. Drugs were supplied by Pfizer and shipped to University Hospitals Bristol Pharmacy who prepared two batches of 36 bottles (one for male and one for females). Within each

187 group of 36 bottles, 18 bottles contained 10 varenicline tablets ( $0.5 \mathrm{mg}$ each) and 18 bottles contained 10 tablets of matched placebo. Each bottle was given a numeric identifier that enabled study staff involved in data collection to be fully blinded to drug condition.

In addition, an experimental collaborator (who had no direct contact with the study participants) prepared a numeric code using random number assignment software to further randomise participants to CBM groups. Randomisation was stratified so that equal numbers of male and female participants $(n=12)$ were allocated across the six experimental cells (drug treatment [2] × CBM group [3]).

Measures and materials

Materials: Stimuli for the CBM and the fMRI cue exposure task comprised

199 full-colour 32 smoking-related pictures and 32 neutral pictures. Smoking-related

200 cues consisted of full-colour pictures of people smoking. Control cues consisted of

201 full-colour pictures of people engaged in everyday activities (e.g., talking on the

202 telephone, writing). Equal numbers of females and males were represented in each category. The set of cues pictures is the same as used in previous imaging studies (McClernon, Kozink, Lutz, \& Rose, 2009; McClernon, Kozink, \& Rose, 2008). For the cognitive bias modification task, an additional 4 picture pairs, unrelated to smoking, were used in practice and buffer trials. 
Running head: Varenicline, cognitive bias modification and $\mathrm{fMRI}$ in smokers

208 modified visual dot probe task designed to induce a biased cognitive response away

209 from (avoid: $n=24$ ), or towards (attend: $n=24$ ) smoking-related cues, or a control

210 condition (control: $\mathrm{n}=24$ ). Each task version comprised 768 trials. Each trial began

211 with a fixation cross (500 ms), before a picture pair (smoking image, neutral image)

212 was presented on a computer screen. The picture pair stayed on screen for $500 \mathrm{~ms}$

213 and then was replaced by a probe (small square or circle) in a location previously

214 occupied by one of the pictures. Participants were required to identity whether the

215 probe was a square or circle by pressing designated keyboard keys.

216 The majority of trials $(n=512)$ were training trials, presented in four blocks,

217 and the remainder of trials $(n=256)$ were test trials. Half of the test trials $(n=128)$

218 were presented prior to the training trials (baseline test), and half $(n=128)$ after the

219 training trials, in order to assess the effect of the CBM on cognitive bias. In the test

220 trials, the probe appeared with equal frequency in the location of the smoking-related

221 or neutral picture. In the training trials, the probe appeared in the location of the

222 neutral picture on $75 \%$ of trials in the avoid condition, or the smoking-related picture

223 on $75 \%$ of trials in the attend condition, or with equal frequency in the location of

224 neutral and smoking-related pictures in the control condition. The inter-trial interval

225 jittered between $750 \mathrm{~ms}$ and 1,250 ms. The tasks were programmed and presented

226 using EPrime version 2 software (Psychology Software Tools Inc., Pittsburgh PA),

227 and total task time was approximately 50 minutes.

Cognitive Bias Generalisation Test (modified Stroop): A pictorial version of the

modified Stroop task was used to investigate the effect of dot-probe CBM on a different

230 measure of cognitive bias. The task began with 16 practice trials followed by two

231 experimental blocks, each comprising 8 buffer and 96 experimental trials (i.e., 208

232 trials in total). For each trial a picture was presented (smoking-related or neutral)

233 centrally on screen. The picture was surrounded by a coloured border and the 
Running head: Varenicline, cognitive bias modification and fMRI in smokers

234 participant was required to identify the colour of the border (red, blue, yellow or green)

235 using colour-marked keys on the keyboard.

236 Questionnaires: Questionnaire measures included the Eysenck Personality

237 Questionnaire - Revised (EPQ-R) (Eysenck \& Eysenck, 1991), the Questionnaire of

238 Smoking Urges - Brief (QSU-Brief) (Tiffany \& Drobes, 1991), the Minnesota Nicotine

239 Withdrawal Scale (MNWS) (Hughes \& Hatsukami, 1986) and visual analogue scales

240 (VAS) of mood and cigarette craving.

241 fMRI Acquisition: An anatomical and a fMRI scan were performed on the test

242 day (Day 7) with a Siemens Magnetom Skyra 3T scanner. BOLD images were

243 acquired using an EPI sequence (36 slices, TR $=2,500 \mathrm{~ms}$, TE $=30 \mathrm{~ms}$, FOV $=19.2$

$244 \mathrm{~cm}$, matrix $=64 \times 64$, flip angle $=90^{\circ}$, slice thickness $=3 \mathrm{~mm}$, resulting in $3 \mathrm{~mm}^{3}$

245 isotropic voxels). A T1-weighted structural image was acquired using an MP-RAGE

246 sequence with a $0.9 \mathrm{~mm}^{3}$ isotropic voxel size and 192 slices. During the $\mathrm{fMRI}$ cue

247 exposure procedure, smoking-related and control cues were presented in a boxcar

248 design with four blocks per category. Participants were required to make a button

249 press on each stimulus presentation to confirm they had seen the image (this did not

250 terminate viewing time). Each block lasted $40 \mathrm{~s}$, during which time 8 cues were

251 presented. Before and after each block, a crosshair was presented for $5 \mathrm{~s}$.

252 Participants were then asked to rate cigarette craving on an 8-point scale ("none at

253 all" to "extreme"). The scale was presented for $10 \mathrm{~s}$ followed by a crosshair for

254 another $10 \mathrm{~s}$. Thus, the total interblock-interval was $25 \mathrm{~s}$. The sequence of events

255 was controlled using EPrime version 2 software (Psychology Software Tools Inc.,

256 Pittsburgh PA), and total task time was approximately $10 \mathrm{~min}$.

Procedure

Individuals who responded to study advertisements were sent the full

260 information sheet and completed a telephone screening to assess basic eligibility.

261 Eligible participants were then booked in for a screening and baseline assessment 
Running head: Varenicline, cognitive bias modification and fMRI in smokers

262

263

264

265

266

267

268

269

270

271

272

273

274

275

session (Day 0). At this session full written informed consent was taken by a trained researcher, and then the screening procedure was completed. This included measures of expired breath alcohol and carbon monoxide, height, weight, blood pressure and heart rate. A urine screen was performed to test for recent drug use (all) and pregnancy (females). A medical doctor then completed a general physical and psychiatric health assessment, and prescribed the study medication if appropriate. Then participants completed a baseline assessment of cognitive bias (modified Stroop), questionnaires assessing personality (EPQ-R), cigarette craving and withdrawal (QSU, MNWS) and mood (VASs), and a practice version of the task that they would completed during the fMRI scan at the second visit (Day 7).

Participants were then sent away with the study medication, medication packaging information and a drug diary (which they were required to complete and return at the next visit). The second session (test day) was then scheduled for approximately one week later. This session fell on day 7 of their drug regimen.

On the test day (Day 7), participants returned with their drug diaries and any untaken medication. Prior to the scan, they completed the Stroop task followed by a short visual dot probe task that measured baseline cognitive bias. Participants then completed one version of CBM (avoid, attend, control) per the study randomisation. The test version of the dot-probe task was run again immediately post-CBM in order to assess changes in cognitive bias. Following this, participants completed a 4minute anatomical scan and then the cue-exposure test during a 15 minute $\mathrm{fMRI}$ scan. After scanning, participants completed the modified Stroop task and questionnaires (QSU, visual analogue scales) again. At the end of the test session, participants were offered smoking cessation literature, debriefed, and reimbursed.

\section{Data analysis}

The protocol for this study was published in Trials in October 2014 (Attwood, Williams, Adams, McClernon, \& Munafo, 2014). 
Running head: Varenicline, cognitive bias modification and $\mathrm{fMRI}$ in smokers

291 were examined for outliers (defined as scores three or more standard deviations

292 above or below the group mean). Removals are noted in text. Data were assessed

293 for normality and transformed using log 10 transformations (or square root where

data include zero scores) where deviations from normality were observed.

Mean reaction time data were extracted for each of the four variables from the visual dot probe tests that were completed before and after CBM training (pretraining neutral, pre-training smoking, post-training neutral, post-training smoking). Cognitive bias scores were calculated by subtracting RTs to probes that replaced smoking-related pictures from RTs to probes that replaced neutral pictures, so that positive scores represent a bias towards smoking cues and negative scores represent a bias towards neutral cues. These bias scores were used to examine cognitive training effects in a 2 (pre-, post-CBM) $\times 2$ (varenicline, placebo) $\times 3$

303 (attend, avoid, control) mixed model ANOVA. generalisation). Mean reaction times and errors were extracted for smoking and neutral images during tasks completed before and after CBM training. Bias scores were calculated for each variable and used in the same 3-way mixed model ANOVA

308 (detailed above), with exception that the subtraction was reversed (i.e., neutral scores were subtracted from smoking scores). This was done for ease of

310 interpretation as (unlike the visual dot probe) slower scores represent cognitive bias

311 on the Stroop task. Therefore, for Stroop data presented here positive scores

312 represent a cognitive bias to smoking cues.

313 Questionnaire analyses: Withdrawal (MNWS), craving (QSU) and mood (VAS)

314 data were analysed in two time phase analyses using ANOVA. We first assessed tonic 315 craving (QSU) defined as craving during drug treatment. This analysis used baseline 316 data from sessions one and two in a 2 (pre-drug, post-drug treatment) $\times 2$ (varenicline, 317 placebo) mixed model ANOVA. We then examined craving and mood change across 
Running head: Varenicline, cognitive bias modification and $\mathrm{fMRI}$ in smokers

318 CBM (i.e., on test day) in a series of 2 (pre-, post-CBM) $\times 2$ (varenicline, placebo) $\times 3$

319 (attend, avoid, control) mixed ANOVAs.

$320 \quad f M R I$ analysis: BOLD signal pre-processing was conducted in FSL version

3215.0 .1 (Jenkison et al, 2012) to remove noise and artefacts. The first two volumes of each run were discarded to allow for T1 stabilization. All functional images were corrected for head motion using rigid-body transformations (MCFLIRT; Jenkinson et al, 2002) and acquisition timing. Additional pre-processing steps included spatial smoothing using an $8 \mathrm{~mm}$ FWHM Gaussian filter, high-pass filtering, and registration to standard space using FLIRT.

Each participant's fMRI data was then entered into a first-level voxel-by-voxel analysis using the general linear model. Each cue block (smoke, control) was modelled as a boxcar function convolved with a double-y hemodynamic response

330 function that begins at the onset of the first cue in the block and ends at the end of

331 the block (duration $=40 \mathrm{~s}$ ). A smoking $>$ control cue contrast image was created and

332 input into a random effects analysis. A 2 (varenicline, placebo) $\times 3$ (attend, avoid,

333 control) mixed-model whole-brain ANOVA was used to examine smoking cue

334 reactivity (smoking greater than control) between each group. Activation was evaluated within an a priori mask of anatomical brain regions identified in a metaanalysis of cue reactivity (Tang, Fellows, Small, \& Dagher, 2012): nucleus accumbens, caudate, putamen, temporal gyrus, anterior cingulate gyrus, amygdala, insula, posterior cingulate cortex, inferior frontal gyrus, and angular gyrus. Resulting activations within the mask were considered significant at $p<0.001$ (uncorrected)

340 with a minimum cluster extent threshold of 20 contiguous voxels. Smoking cue

341 greater than control cue contrast images for each participant were input into random

342 effects regression analyses examining relations between post-training cognitive bias scores and brain cue reactivity. 
Running head: Varenicline, cognitive bias modification and fMRI in smokers

346 studies (Attwood et al., 2008) indicated a likely increase in cognitive bias index of 30

$347 \mathrm{~ms}$ in the attend group, and a decrease of $30 \mathrm{~ms}$ in the avoid group. We assumed that

348 the change in the control condition would be intermediate (i.e., $0 \mathrm{~ms}$ ). Using these

349 estimates, we calculated that we would achieve greater than $80 \%$ power to detect a

350 linear effect across the three study groups on change in cognitive bias index with a

351 total sample size of $n=30$, at an alpha level of 0.05 . Due to an additional factor of drug

352 group (varenicline vs. placebo), the actual sample size we recruited was $n=72$, with $n$

$353=12$ per experimental group.

354

\section{Results}

\section{Characteristics of participants}

Four participants withdrew from the study (two from varenicline/control CBM condition, and two from placebo/avoid CBM condition) and therefore the final sample comprised 68 participants (53\% female). Participants were aged between 18 and 39

360 years $(M=23, S D=5)$ and smoked between 10 and 25 cigarettes per day $(M=15, S D$

$361=3)$. Alcohol Use Disorders Identifier Test scores ranged between 5 and $24(M=13$,

$362 S D=4)$. EPQ-R scores ranged between 3 and $17(M=8, S D=3)$ for psychoticism, 0

363 and $20(M=7, S D=4)$ for neuroticism, and 10 and $23(M=18, S D=3)$ for extraversion. See Table 1. for participant characteristics by drug and CBM condition. 
Running head: Varenicline, cognitive bias modification and fMRI in smokers

Table 1. Mean $(S D)$ participant characteristics, baseline craving and nicotine withdrawal

\begin{tabular}{|c|c|c|c|c|c|c|}
\hline & \multicolumn{2}{|c|}{$\begin{array}{c}\text { Attend } \\
(\mathrm{N}=24)\end{array}$} & \multicolumn{2}{|c|}{$\begin{array}{c}\text { Avoid } \\
(\mathrm{N}=22)\end{array}$} & \multicolumn{2}{|c|}{$\begin{array}{c}\text { Control } \\
(\mathrm{N}=22)\end{array}$} \\
\hline & $\begin{array}{l}\text { Varen. } \\
\qquad(\mathrm{N}=12)\end{array}$ & $\begin{array}{c}\text { Placebo } \\
(\mathrm{N}=12)\end{array}$ & $\begin{array}{l}\text { Varen. } \\
(\mathrm{N}=12)\end{array}$ & $\begin{array}{l}\text { Placebo } \\
(\mathrm{N}=10)\end{array}$ & $\begin{array}{l}\text { Varen. } \\
(N=10)\end{array}$ & $\begin{array}{l}\text { Placebo } \\
(\mathrm{N}=12)\end{array}$ \\
\hline Age & $\begin{array}{l}25.5 \\
(4.9)\end{array}$ & $\begin{array}{l}22.3 \\
(4.5)\end{array}$ & $\begin{array}{l}26.0 \\
(7.6)\end{array}$ & $\begin{array}{l}21.7 \\
(3.0)\end{array}$ & $\begin{array}{l}21.9 \\
(3.3)\end{array}$ & $\begin{array}{l}21.3 \\
(2.9)\end{array}$ \\
\hline CPD & $\begin{array}{l}15.5 \\
(2.8)\end{array}$ & $\begin{array}{l}14.5 \\
(3.7)\end{array}$ & $\begin{array}{l}15.5 \\
(3.6)\end{array}$ & $\begin{array}{l}14.2 \\
(2.9)\end{array}$ & $\begin{array}{l}14.6 \\
(3.3)\end{array}$ & $\begin{array}{l}16.1 \\
(3.6)\end{array}$ \\
\hline AUDIT & $\begin{array}{c}8.1 \\
(5.1)\end{array}$ & $\begin{array}{c}9.3 \\
(3.9)\end{array}$ & $\begin{array}{c}9.5 \\
(3.3)\end{array}$ & $\begin{array}{c}9.0 \\
(2.9)\end{array}$ & $\begin{array}{c}9.5 \\
(3.2)\end{array}$ & $\begin{array}{c}8.0 \\
(3.0)\end{array}$ \\
\hline $\begin{array}{c}\text { Baseline } \\
\text { QSU }\end{array}$ & $\begin{array}{l}35.4 \\
(8.7)\end{array}$ & $\begin{array}{c}35.7 \\
(11.0)\end{array}$ & $\begin{array}{c}33.8 \\
(12.3)\end{array}$ & $\begin{array}{c}38.2 \\
(10.6)\end{array}$ & $\begin{array}{l}31.5 \\
(9.8)\end{array}$ & $\begin{array}{l}36.4 \\
(9.8)\end{array}$ \\
\hline $\begin{array}{c}\text { Baseline } \\
\text { MNWS }\end{array}$ & $\begin{array}{c}7.2 \\
(5.5)\end{array}$ & $\begin{array}{c}8.5 \\
(6.9)\end{array}$ & $\begin{array}{c}6.6 \\
(3.9)\end{array}$ & $\begin{array}{c}9.9 \\
(9.6)\end{array}$ & $\begin{array}{c}5.8 \\
(4.9)\end{array}$ & $\begin{array}{l}10.5 \\
(6.8)\end{array}$ \\
\hline $\begin{array}{c}\text { EPQ-R } \\
\text { Neu. }\end{array}$ & $\begin{array}{c}7.1 \\
(4.7)\end{array}$ & $\begin{array}{c}7.0 \\
(3.5)\end{array}$ & $\begin{array}{c}7.4 \\
(4.8)\end{array}$ & $\begin{array}{c}7.0 \\
(5.5)\end{array}$ & $\begin{array}{c}5.0 \\
(2.9)\end{array}$ & $\begin{array}{c}6.4 \\
(4.6)\end{array}$ \\
\hline $\begin{array}{c}\text { EPQ-R } \\
\text { Psy. }\end{array}$ & $\begin{array}{c}8.3 \\
(4.2)\end{array}$ & $\begin{array}{c}6.7 \\
(2.8)\end{array}$ & $\begin{array}{c}7.4 \\
(3.6)\end{array}$ & $\begin{array}{c}6.1 \\
(1.9)\end{array}$ & $\begin{array}{c}7.4 \\
(3.1)\end{array}$ & $\begin{array}{c}9.0 \\
(4.1)\end{array}$ \\
\hline $\begin{array}{c}\text { EPQ-R } \\
\text { Ext. }\end{array}$ & $\begin{array}{l}17.9 \\
(3.4)\end{array}$ & $\begin{array}{l}18.8 \\
(3.1)\end{array}$ & $\begin{array}{l}17.9 \\
(2.7)\end{array}$ & $\begin{array}{l}17.5 \\
(3.3)\end{array}$ & $\begin{array}{l}17.7 \\
(2.4)\end{array}$ & $\begin{array}{l}17.8 \\
(2.7)\end{array}$ \\
\hline
\end{tabular}

370

Values represent mean (SD). SD = standard deviation; Varen. $=$ varenicline, $\mathrm{CPD}=$ cigarettes per day; AUDIT = Alcohol Use Disorder Identifier Test; QSU = Questionnaire of smoking urges; MNWS = Minnesota Nicotine Withdrawal Scale; EPQ-R = Eysenck Personality QuestionnaireRevised, $\mathrm{Neu}=$ neuroticism; Psy = psychoticism; Ext $=$ extraversion .

\section{Cognitive bias modification test (visual dot probe)}

Due to computer malfunction, post-training CBM data were not recorded for one participant, therefore post-training sample comprises 67 participants. This participant completed the allocated CBM training and therefore their data have been retained in all other analysis. Prior to the calculation of bias scores, reaction time data of the four primary variables (pre-training neutral, pre-training smoking, post-training neutral, post-training smoking) were assessed for normality, and there was evidence of positive skewness on three of the four variables. Therefore log 10 transformation was 
Running head: Varenicline, cognitive bias modification and fMRI in smokers

384

385

386

387

388

389

390

391

392

393

394

395

396

397

398

399

400

401

402

403

404

405

406

407

408

409

410

411

applied to the data prior to the bias calculations being performed. The 2 (pre-, post-

CBM) $\times 2$ (varenicline, placebo) $\times 3$ (attend, avoid, control) mixed model ANOVA showed no main effects or interactions ( $p s>.23$ ). These findings did not change if untransformed data were used ( $p s>.18$ ).

\section{Cognitive bias generalisation test (modified Stroop)}

For error data, three participants were identified as outliers in the pre-CBM condition and one participant was identified as an outlier in the post-CBM condition.

These data were removed from main analysis. After data removal error data were not normally distributed and a square root transformation was applied to these data.

There was weak evidence of a drug $\times$ CBM interaction $(F[1,57]=2.74, p=$ $\left..073, \eta_{p}^{2}=.09\right)$ for errors, reflecting a bias towards smoking (versus neutral) cues in the attend CBM condition (compared to avoid and neutral conditions) but only in individuals who had received placebo. In contrast, participants who received varenicline showed a bias towards neutral in the attend condition (compared to avoid and neutral conditions). The evidence for this effect was weaker when untransformed data were used $(p=.18)$ and when outliers were included $(p=.29)$. There was no evidence of any other main effects or interactions for Stroop error ( $p s>.13$ ) or reaction time (ps >.15) data.

\section{Questionnaire data}

Withdrawal across drug treatment: There was weak evidence of an effect of $\operatorname{drug}\left(F[1,66]=3.34, p=.072, \eta_{p}^{2}=.05\right)$ with lower nicotine withdrawal in the drug $(M$ $=7.0, S D=6.3)$ compared to placebo group $(M=9.8, S D=6.3)$. There was no clear evidence of an effect of time or time by drug interaction ( $p s>.35$ ).

Tonic craving across drug treatment: For QSU data, there was evidence of effects of time $\left(F[1,65]=33.61, p<.001, \eta_{p}^{2}=.34\right)$ and drug $(F[1,65]=6.06, p=.017$, $\left.\eta_{p}^{2}=.09\right)$, which were subsumed under a time $\times$ drug interaction $(F[1,65]=4.37, p=$ 
Running head: Varenicline, cognitive bias modification and fMRI in smokers

$\left.4120.04, \eta_{p}^{2}=.06\right)$. Post-hoc paired samples t-tests showed that there was a decrease in

413 craving from session one (pre-drug) to session two (post-drug) in both drug groups, but

414 this effect was larger in the varenicline group (see Table 2). For cigarette craving VAS

415 data, the main effects of time $\left(F[1,61]=56.62, p<.001, \eta_{p}^{2}=.48\right)$ and $\operatorname{drug}(F[1,61]=$

$41616.32, p<.001, \eta_{p}{ }^{2}=.21$ ) were replicated but there were no other effects or

417 interactions ( $p s>.34)$.

Mood (VAS) across drug treatment: There was evidence of effects of time for

happiness $\left(F[1,62]=4.37, p=.041, \eta_{p}^{2}=.07\right)$, drowsiness $(F[1,62]=3.78, p=.057$,

$\left.\eta_{p}^{2}=.06\right)$, depression $\left(F[1,62]=9.93, p=.003, \eta_{p}^{2}=.14\right)$, anxiety $(F[1,62]=3.22, p=$

$\left..078, \eta_{p}^{2}=.05\right)$ and irritability $\left(F[1,62]=8.56, p=.005 \eta_{p}^{2}=.12\right)$, with decreases in

also evidence of an effect of drug for anxiety $\left(F[1,62]=9.01, p=.004, \eta_{p}^{2}=.13\right)$, with

lower anxiety reported in the varenicline group. There was no clear evidence of any other main effects or interactions ( $p s>.10)$.

Table 2: Craving (QSU) scores from pre- to post drug administration in varenicline and placebo groups

\begin{tabular}{l|c|c|c|c|} 
& $\begin{array}{c}\text { Mean difference } \\
\text { from session one } \\
\text { to session two } \\
(\text { SD) }\end{array}$ & $\begin{array}{c}\text { Effect size } \\
(d z)\end{array}$ & $95 \% \mathrm{Cl}$ & $p$-value \\
\hline Placebo & $-4.7(10.3)$ & 0.46 & -8.4 to -1.1 & 0.013 \\
\hline Varenicline & $-10.1(10.6)$ & 0.95 & -13.8 to -6.4 & $<0.001$ \\
\hline
\end{tabular}

Withdrawal across CBM (pre-CBM to post-scan): There was evidence of a main effect of time $\left(F[1,62]=14.98, p<.001, \eta_{p}^{2}=.20\right)$ with increases in withdrawal pre-CBM $(M$ $=8.6, S D=7.3)$ to post-CBM $(M=11.3, S D=7.7)$. There was no clear evidence for other main effects or interactions ( $p s>.14)$. 
Running head: Varenicline, cognitive bias modification and fMRI in smokers

436

437

Craving (QSU) across CBM (pre-CBM to post-scan): For QSU data, there was evidence of effects of time $\left(F[1,62]=62.6, p<.001, \eta_{p}^{2}=.50\right)$ and drug $(F[1,62]=$ 8.82, $\left.p=.004, \eta_{p}^{2}=.13\right)$, indicating increases in craving from pre-CBM $(M=27.7, S D=$ $12.1)$ to post $\operatorname{scan}(M=38.5, S D=12.8)$ and higher craving in the placebo group $(M=$ $36.9, S D=9.7)$ compared to varenicline group $(M=29.3, S D=11.6)$. There was no strong evidence of other main effects or interactions ( $p s>.08)$. The main effects of time $\left(F[1,62]=56.62, p<.001, \eta_{p}^{2}=.48\right)$ and drug $\left(F[1,62]=16.32, p<.001, \eta_{p}{ }^{2}=\right.$ .21) were replicated using craving VAS data, and there was no clear evidence of any other effects or interactions ( $p s>34$ ).

Mood across CBM session (pre-CBM to post-scan): There was evidence of main effects of time for happiness $\left(F(1,61)=4.24, p=.044, \eta_{p}^{2}=.07\right)$, drowsiness $\left(F(1,61)=12.86, p=.001, \eta_{p}^{2}=.17\right)$, energy $\left(F(1,61)=8.24, p=.006, \eta_{p}^{2}=.12\right)$ and irritability $\left(F(1,61)=7.71, p=.007, \eta_{p}^{2}=.11\right)$, with decreases in happiness and energy, and increases in drowsiness and irritability across the session. There was evidence of a main effect of drug $\left(F(1,61)=6.46, p=.014, \eta_{p}^{2}=.10\right)$ and a time $\times$ drug interaction $\left(F(1,61)=6.15, p=.016, \eta_{p}^{2}=.09\right)$ for anxiety, with higher anxiety in the placebo group $(M=25.4, S D=19.9)$ compared to varenicline group $(M=15.1, S D=14.0)$. Post-hoc paired t-tests indicated a decrease in anxiety across the session in the placebo group $(t=2.35, d f=33, p=.025, d z=.40)$, but not the varenicline group $(t=-1.25, d f=32, p$ $=.22, d z=.22)$. Finally there was evidence of a drug $\times$ CBM interaction for happiness $\left(F(2,61)=4.36, p=.017, \eta_{p}^{2}=.13\right)$, with the varenicline group reporting lower happiness than the placebo group but only in the attend CBM condition.

\section{Neural response (fMRI data)}

Following pre-processing, three participants ( 2 in the varenicline-attend group,

1 in the control-placebo group) were excluded from fMRI imaging analysis ( 2 due to excessive motion and 1 due to poor signal quality). There was no evidence of main effects of drug or CBM within the a priori mask. There was strong evidence of a drug $\times$ 
Running head: Varenicline, cognitive bias modification and fMRI in smokers

464 CBM interaction in one cluster $(\mathrm{k}=32)$ in the right middle temporal gyrus (peak voxel: $\mathrm{x}$

$465=58, y=-58, z=12 ; z=3.78, p<0.001)$. The placebo group exhibited greater

466 temporal gyrus activation to smoking cues than the varenicline group in the control

467 CBM condition $(\mathrm{t}=2.86, p=.006)$ but less activation than the varenicline group in the toward CBM condition $(\mathrm{t}=2.41, p=.02)$ (Figure 1$)$.

469

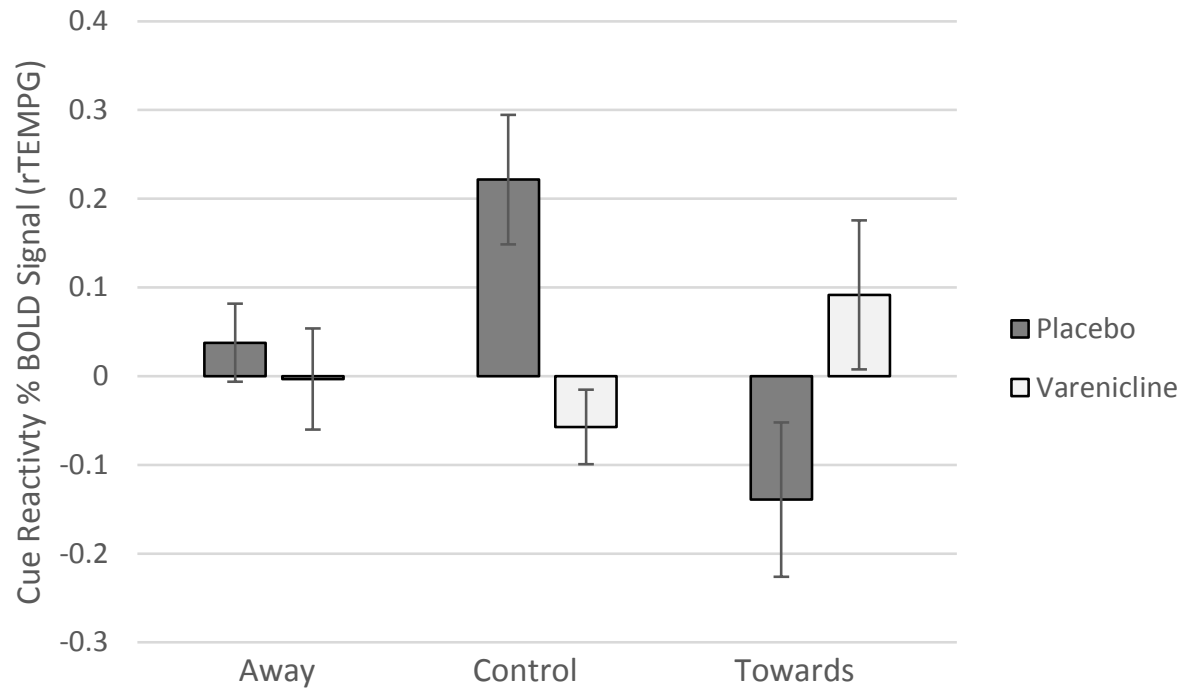

470

471

Figure 1: Neural response to smoking cues (\% BOLD signal) in the right temporal gyrus across the three CBM groups following 1-week administration of placebo or varenicline. analysis were transformed as described above. One subject with useable imaging data was excluded from the analysis due to incomplete behavioural data. Correlation

479 between post-training bias scores and smoking cue reactivity (i.e., smoking greater 480 than control cue contrasts) were put into a regression analysis. There was no clear 481 evidence for areas of activation ( $p s>0.001$ ). 
Running head: Varenicline, cognitive bias modification and $\mathrm{fMRI}$ in smokers

\section{Discussion}

We investigated the effects of CBM and one-week varenicline treatment on neural response to smoking-related cues in current smokers. There was no evidence that CBM training alone altered cognitive bias (post-CBM visual dot probe, Stroop), mood, craving or neural response to smoking-cues. There was evidence of reduced craving in the varenicline group as evinced by several drug by time interactions. Across the drug administration phase of the study (i.e., the six days preceding the study session), there was a greater reduction in QSU scores for the varenicline compared to placebo group. A main effect of drug was also evident for VAS craving scores (i.e., lower craving in varenicline group); however, the drug by time interaction was not replicated. The lower reports of craving in the varenicline group were also evident at the study session, during which CBM was administered. Finally, there was weak evidence that varenicline may have attenuated CBM-induced smoking bias, as there was an increase in smoking bias following CBM attend training, but only in the placebo group. There was no evidence of an interaction between varenicline and CBM on withdrawal, craving or mood.

There was evidence of a drug by CBM interaction on neural responses in one region within our a priori cue-reactivity mask. In right middle temporal gyrus (rMTG), activation in response to smoking relative to neutral cues was greater in the placebo as compared to the varenicline condition in the control CBM condition; the opposite pattern was observed in the toward CBM condition. Whereas the rTMG has been shown to be active in response to viewing smoking cues in meta-analyses (Tang et al., 2012), little has been reported regarding its potential role in processing conditioned smoking cues. Activation in the specific rTMG location we observed, has also been observed in studies of Theory of Mind, or the social-cognitive ability to infer the emotional and motivational experience (Vollm et al., 2006), where it may be involved in retrieval of memories associated with the behaviour of others. In the context of the present study, varenicline in the control CBM condition may decrease the degree to 
Running head: Varenicline, cognitive bias modification and fMRI in smokers

511 which such memories are accessed due to decreased tonic craving. Why toward CBM

512 training would reverse such effects is unclear from the data, which suggests that

513 additional research is needed to fully understand the influence of CBM on the

514 processing of smoking cues.

515 Taken together these findings support a benefit of varenicline on tonic craving

516 and neural response to smoking cues (which may be driven by the craving effects).

517 While the effects of varenicline may be small, they are meaningful given the fact that

518 the dosing regime delivered in the study is substantially lower than the clinically

519 prescribed dose (i.e., 1 week compared to a standard 12-week course). However, we

520 found no evidence of a benefit of CBM on any outcomes, and little evidence that

521 varenicline would be a useful adjunct to smoking-related CBM. The CBM by drug

522 interaction that was observed for the fMRI data, indicated that the effects of varenicline

523 may have been attenuated for active CBM (i.e., the effects were only observed in the

524 control training group). However, numbers are small and therefore this effect requires

525 replication.

526 It is noteworthy that we did not find effects of CBM on measures of cognitive

527 bias (visual dot probe and Stroop). There are known issues with the reliability of

528 cognitive bias tests (Ataya et al., 2012), and therefore this may be a failure of the

529 measure rather than a lack of effect. However, this indicates that the CBM may not

530 have been effective, and these findings should be interpreted with this in mind. We

531 hypothesised that effects of CBM would be potentiated by varenicline and our failure to

532 observe such effects may be due to there being no CBM effects to strengthen. It is

533 plausible that varenicline may potential effects of CBM if these effects can be reliably

534 achieved.

There are some limitations of this study that should be considered when

536 interpreting these findings. First, our sample size was small for the analysis of

537 interactions. Our planned recruitment of 72 participants was achieved but not all

538 participants were tested to completion, and our final sample was lower ( $n=67$ for 
Running head: Varenicline, cognitive bias modification and fMRI in smokers

539 subjective and cognitive data; $n=64$ for $\mathrm{fMRI}$ data). We also have a computer

540 malfunction for one of the conditions that was not identified until data were extracted.

541 We had to replace a number of participants in one CBM condition (avoid) and therefore

542 these individuals were tested outside of the randomisation sequence. We do not

543 however expect that this had a substantial effect on outcomes as these individuals

544 were testing in close time proximity to the rest of the sample. Furthermore, the

545 researchers collecting data were not aware of the reason for additional recruitment,

546 and therefore double-blinding was maintained. Third, our study recruited non-treatment

547 seeking smokers, and it is plausible that effects of CBM may be stronger in individuals

548 seeking treatment.

549 This study investigated neural responses to smoking cues following varenicline

550 and CBM treatment. There was little evidence of neural effects of either drug or CBM.

551 However, there was evidence of reductions in craving among smokers who completed

552 one-week of varenicline treatment. Drug by CBM interactions were exploratory due to

553 small sample sizes, but we observed an interaction on right temporal gyrus activity.

554 Specifically, varenicline appeared to attenuate cue-related activity in the right temporal

555 gyrus that was presented in the placebo group. However, this effect should be

556 replicated in future research. In summary, this study finds little evidence of clinical

557 potential of CBM.

558

559 
Running head: Varenicline, cognitive bias modification and fMRI in smokers

\section{Abbreviations}

561 CBM: Cognitive bias modification; EPQ-R: Eysenck Personality Questionnaire-

562 Revised; MNWS: Minnesota Nicotine Withdrawal Scale; RT: reaction time: VAS: visual

563 analogue scales.

564

565 Competing interests

566 MRM and ASA have received grant funding from Pfizer Ltd. FJM received partial salary

567 support (2.5\%) from an unrestricted grant funded by Philip Morris from April 2009 to

568 October 2010.

569

570 Author contributions

571 MRM, FJM, SA and ASA contributed to the conception and design of the trial, and plans for data analysis. SA and ASA participated in data collection and project management. FJM and RK analysed and interpreted fMRI data. TW leads the clinical

574 team (including DC, AG and KS) for subject recruitment. ASA drafted the manuscript, 575 and all authors discussed, read and revised the manuscript. All authors approved the 576 publication of this protocol.

578 Acknowledgements

579 This study was supported by a Pfizer Ltd. Investigator Initiated Grant (grant ID:

580 WS676950). MRM, ASA and SA are members of the UK Centre for Tobacco and

581 Alcohol Studies, a UKCRC Public Health Research Centre of Excellence. ASA and

$582 \mathrm{MRM}$ are members of the MRC Integrative Epidemiology Unit at the University of

583 Bristol. We thank Aileen Wilson, Emma Howell, Alexander Davidson, George Stothart and Olivia Maynard for their assistance with data collection. 
bioRxiv preprint doi: https://doi.org/10.1101/480566; this version posted December 21,2018 . The copyright holder for this preprint (which was not certified by peer review) is the author/funder, who has granted bioRxiv a license to display the preprint in perpetuity. It is made available under aCC-BY 4.0 International license.

Running head: Varenicline, cognitive bias modification and fMRI in smokers

586

587

588

589

590

591

592

593

594

595

596

597

598

599

600

601

602

603

604

605

606

607

608

609

610

611

612

613

614

615

616

617

618

619

620

621

622

623

624

625

626

627

628

629

630

631

632

633

634

635

636

637

638

639

\section{References}

Abrams, D. B., Monti, P. M., Carey, K. B., Pinto, R. P., \& Jacobus, S. I. (1988). Reactivity to smoking cues and relapse: two studies of discriminant validity. Behav Res Ther, 26(3), 225-233. doi:0005-7967(88)90003-4 [pii]

Ataya, A. F., Adams, S., Mullings, E., Cooper, R. M., Attwood, A. S., \& Munafo, M. R. (2012). Internal reliability of measures of substance-related cognitive bias. Drug Alcohol Depend, 121(1-2), 148-151. doi:10.1016/j.drugalcdep.2011.08.023

Attwood, A. S., O'Sullivan, H., Leonards, U., Mackintosh, B., \& Munafo, M. R. (2008). Attentional bias training and cue reactivity in cigarette smokers. Addiction, 103(11), 1875-1882. doi:10.1111/j.1360-0443.2008.02335.x

Attwood, A. S., Williams, T., Adams, S., McClernon, F. J., \& Munafo, M. R. (2014). Effects of varenicline and cognitive bias modification on neural response to smoking-related cues: study protocol for a randomized controlled study. Trials, 15, 391. doi:10.1186/1745-6215-15-391

Baert, S., De Raedt, R., Schacht, R., \& Koster, E. H. (2010). Attentional bias training in depression: therapeutic effects depend on depression severity. J Behav Ther Exp Psychiatry., 41(3), 265-274. doi:10.1016/j.jbtep.2010.02.004

Brandon, T. H., Drobes, D. J., Unrod, M., Heckman, B. W., Oliver, J. A., Roetzheim, R. C., ... Small, B. J. (2011). Varenicline effects on craving, cue reactivity, and smoking reward. Psychopharm. (Berl), 218(2), 391-403. doi:10.1007/s00213011-2327-z

Cox, W. M., Pothos, E. M., \& Hosier, S. G. (2007). Cognitive-motivational predictors of excessive drinkers' success in changing. Psychopharm. (Berl), 192(4), 499510. doi:10.1007/s00213-007-0736-9

Eysenck, H. J., \& Eysenck, S. B. G. (1991). The Eysenck Personality Questionnaire Revised. London: Hodder and Stoughton.

Field, M., \& Christiansen, P. (2012). Commentary on, 'Internal reliability of measures of substance-related cognitive bias'. Drug Alcohol Depend, 124(3), 189-190. doi:10.1016/j.drugalcdep.2012.02.009

Field, M., Duka, T., Tyler, E., \& Schoenmakers, T. (2009). Attentional bias modification in tobacco smokers. Nicotine Tob Res, 11(7), 812-822. doi:10.1093/ntr/ntp067

Field, M., Munafo, M. R., \& Franken, I. H. (2009). A meta-analytic investigation of the relationship between attentional bias and subjective craving in substance abuse. Psychol Bull, 135(4), 589-607. doi:2009-09537-005 [pii]

$10.1037 / \mathrm{a} 0015843$

Foltin, R. W., \& Haney, M. (2000). Conditioned effects of environmental stimuli paired with smoked cocaine in humans. Psychopharm. (Berl), 149(1), 24-33.

Franken, I. H. (2003). Drug craving and addiction: integrating psychological and neuropsychopharmacological approaches. Prog Neuropsychopharmacol Biol Psychiatry, 27(4), 563-579. doi:S0278-5846(03)00081-2 [pii]

10.1016/S0278-5846(03)00081-2

Gray, K. M., LaRowe, S. D., \& Upadhyaya, H. P. (2008). Cue reactivity in young marijuana smokers: a preliminary investigation. Psychol Addict Behav, 22(4), 582-586. doi:2008-17215-015 [pii]

$10.1037 / \mathrm{a} 0012985$

Hester, R., \& Luijten, M. (2013). Neural correlates of attentional bias in addiction. CNS Spectr., 1-8. doi:10.1017/S1092852913000473

Hughes, J. R., \& Hatsukami, D. (1986). Signs and symptoms of tobacco withdrawal. Arch Gen Psychiatry, 43(3), 289-294.

Le Foll, B., Chakraborty-Chatterjee, M., Lev-Ran, S., Barnes, C., Pushparaj, A., Gamaleddin, I., ... Goldberg, S. R. (2012). Varenicline decreases nicotine selfadministration and cue-induced reinstatement of nicotine-seeking behaviour in rats when a long pretreatment time is used. Int $J$ Neuropsychopharmacol., 15(9), 1265-1274. doi:10.1017/S1461145711001398 
bioRxiv preprint doi: https://doi.org/10.1101/480566; this version posted December 21,2018 . The copyright holder for this preprint (which was not certified by peer review) is the author/funder, who has granted bioRxiv a license to display the preprint in perpetuity. It is made available under aCC-BY 4.0 International license.

Running head: Varenicline, cognitive bias modification and $\mathrm{fMRI}$ in smokers

640

641

642

643

644

645

646

647

648

649

650

651

652

653

654

655

656

657

658

659

660

661

662

663

664

665

666

667

668

669

670

671

672

673

674

675

676

677

678

679

680

681

682

683

684

685

686

687

688

689

690

691

692

693

694
Lopes, F. M., Pires, A. V., \& Bizarro, L. (2014). Attentional bias modification in smokers trying to quit: a longitudinal study about the effects of number of sessions. $J$ Subst Abuse Treat, 47(1), 50-57. doi:10.1016/j.jsat.2014.03.002

Marissen, M. A., Franken, I. H., Blanken, P., van den Brink, W., \& Hendriks, V. M. (2007). Cue exposure therapy for the treatment of opiate addiction: results of a randomized controlled clinical trial. Psychother Psychosom, 76(2), 97-105. doi:000097968 [pii]

$10.1159 / 000097968$

McClernon, F. J., Conklin, C. A., Kozink, R. V., Adcock, R. A., Sweitzer, M. M., Addicott, M. A., . . . DeVito, A. M. (2016). Hippocampal and Insular Response to Smoking-Related Environments: Neuroimaging Evidence for Drug-Context Effects in Nicotine Dependence. Neuropsychopharmacology, 41(3), 877-885. doi:10.1038/npp.2015.214

McClernon, F. J., Kozink, R. V., Lutz, A. M., \& Rose, J. E. (2009). 24-h smoking abstinence potentiates fMRI-BOLD activation to smoking cues in cerebral cortex and dorsal striatum. Psychopharmacology (Berl), 204(1), 25-35. doi:10.1007/s00213-008-1436-9

McClernon, F. J., Kozink, R. V., \& Rose, J. E. (2008). Individual differences in nicotine dependence, withdrawal symptoms, and sex predict transient fMRI-BOLD responses to smoking cues. Neuropsychopharmacology, 33(9), 2148-2157. doi:10.1038/sj.npp.1301618

McHugh, R. K., Murray, H. W., Hearon, B. A., Calkins, A. W., \& Otto, M. W. (2010). Attentional bias and craving in smokers: the impact of a single attentional training session. Nicotine Tob Res, 12(12), 1261-1264. doi:10.1093/ntr/ntq171

Mucha, R. F., Pauli, P., \& Angrilli, A. (1998). Conditioned responses elicited by experimentally produced cues for smoking. Can J Physiol Pharmacol, 76(3), 259-268.

Muntaner, C., Cascella, N. G., Kumor, K. M., Nagoshi, C., Herning, R., \& Jaffe, J. (1989). Placebo responses to cocaine administration in humans: effects of prior administrations and verbal instructions. Psychopharm. (Berl), 99(2), 282-286.

Niaura, R., Abrams, D., Demuth, B., Pinto, R., \& Monti, P. (1989). Responses to smoking-related stimuli and early relapse to smoking. Addict Behav, 14(4), 419428.

Ray, L. A., Lunny, K., Bujarski, S., Moallem, N., Krull, J. L., \& Miotto, K. (2013). The effects of varenicline on stress-induced and cue-induced craving for cigarettes. Drug Alcohol Depend, 131(1-2), 136-142. doi:10.1016/j.drugalcdep.2012.12.015

Swerdlow, N. R. (2012). Beyond antipsychotics: pharmacologically-augmented cognitive therapies (PACTs) for schizophrenia. Neuropsychopharm., 37(1), 310-311. doi:10.1038/npp.2011.195

Tang, D. W., Fellows, L. K., Small, D. M., \& Dagher, A. (2012). Food and drug cues activate similar brain regions: a meta-analysis of functional MRI studies. Physiol Behav, 106(3), 317-324. doi:10.1016/j.physbeh.2012.03.009

Tiffany, S. T., \& Drobes, D. J. (1991). The development and initial validation of a questionnaire on smoking urges. Br J Addict, 86(11), 1467-1476.

Unrod, M., Drobes, D. J., Stasiewicz, P. R., Ditre, J. W., Heckman, B., Miller, R. R., . . Brandon, T. H. (2014). Decline in cue-provoked craving during cue exposure therapy for smoking cessation. Nicotine Tob Res, 16(3), 306-315. doi:10.1093/ntr/ntt145

Vollm, B. A., Taylor, A. N., Richardson, P., Corcoran, R., Stirling, J., McKie, S., . . Elliott, R. (2006). Neuronal correlates of theory of mind and empathy: a functional magnetic resonance imaging study in a nonverbal task. Neuroimage, 29(1), 90-98. doi:10.1016/j.neuroimage.2005.07.022

Wakefield, M., Germain, D., \& Henriksen, L. (2008). The effect of retail cigarette pack displays on impulse purchase. Addiction, 103(2), 322-328. doi:ADD2062 [pii] 
Running head: Varenicline, cognitive bias modification and fMRI in smokers

695

696

697

698

699

700

701

702

703

704

705

706

10.1111/j.1360-0443.2007.02062.x

Waters, A. J., Shiffman, S., Sayette, M. A., Paty, J. A., Gwaltney, C. J., \& Balabanis, M. H. (2004). Cue-provoked craving and nicotine replacement therapy in smoking cessation. J Consult Clin Psychol, 72(6), 1136-1143. doi:10.1037/0022-006X.72.6.1136

World Health Organization. (2013). WHO Global Report: Mortality Attributable to Tobacco. Retrieved from

Wouda, J. A., Riga, D., De Vries, W., Stegeman, M., van Mourik, Y., Schetters, D., . . De Vries, T. J. (2011). Varenicline attenuates cue-induced relapse to alcohol, but not nicotine seeking, while reducing inhibitory response control.

Psychopharmacology (Berl), 216(2), 267-277. doi:10.1007/s00213-011-2213-8 